Meta

Journal des traducteurs

Translators' Journal

\title{
Un bestiaire linguistique - ou les animaux dans les images du français et de l'anglais
}

\section{Henri Van Hoof}

Volume 48, numéro 4, décembre 2003

URI : https://id.erudit.org/iderudit/008726ar

DOI : https://doi.org/10.7202/008726ar

Aller au sommaire du numéro

Éditeur(s)

Les Presses de l'Université de Montréal

ISSN

0026-0452 (imprimé)

1492-1421 (numérique)

Découvrir la revue

Citer ce document

Van Hoof, H. (2003). Un bestiaire linguistique - ou les animaux dans les images du français et de l'anglais. Meta, 48(4), 559-584.

https://doi.org/10.7202/008726ar d'utilisation que vous pouvez consulter en ligne. 


\title{
ÉTUDES TERMINOLOGIQUES ET LINGUISTIQUES
}

\section{Un bestiaire linguistique - ou les animaux dans les images du français et de l'anglais}

\author{
HENRI VAN HOOF \\ Centre de Terminologie de Bruxelles, Bruxelles, Belgique
}

(Suite du volume 47, $n^{\circ} 3$, septembre 2002)

\section{CABRI}

effarouché comme un cabri

très timide

(E) as timid as a hare/rabbit

\section{CACHALOT}

souffler comme un cachalot (19)

(cf. souffler comme un bœuf)

\section{CAFARD}

avoir le cafard/un coup de cafard (1857)

(cf. avoir le bourdon)

cafard

1. cf. bourrique 5 (1834)

2. cf. bourdon 2 (1857)

3. rapporteur (1834)

(E) sneak

\section{CAILLE}

au premier son on ne prend la caille (15)

les femmes aiment se faire prier avant de céder

(E) women do not consent readily

caille (17)

1. cf. bête 2

2. cf. biche 3

caille coiffée (1640)

(cf. araignée de trottoir)

chasser à la caille coiffée (1894)

courir les filles

(E) to look for beaver, to do a grouse, to chase pussy

dodu comme une caille

bien en chair

(E) as plump as a partridge

être chaud comme une caille (1842)

1. avoir chaud

2. être d'un tempérament sensuel
(E) 1. to be hot

2. to be highly sexed

la mère aux cailles (1640)

grosse femme

(E) fat woman

ma petite caille! (1850)

(cf. ma biche!)

on n'a pas la caille pour ha ha crier (1640)

il ne faut pas crier ses intentions sur les toits quand on est engagé dans une entreprise délicate

(E) to scare a bird is not the way to catch it plus la caille carcaille, plus chère est la semaille

les cailles mangeuses de graines sont un danger pour les semaines

(E) quails are a danger at seedtime sourd comme une caille

(cf. sourd comme une bécasse)

\section{CAÏMAN}

caïman (1880)

surveillant général (à l'École normale

supérieure)

(E) chief-master on duty

\section{CALAMAR}

calamar

personne de grande taille

(E) tall person

\section{CAMÉLÉON}

caméléon

1. personne versatile (1678)

2. personne qui vit de peu

3. gros trafiquant de drogue

(E) 1. cameleon (1975)

2. one who lives on thin air

3. big drug dealer 


\section{CANARD}

avoir couvé un canard

avoir un enfant très différent du reste de la famille

(E) to have a child completely different from the rest of the family

bailler/donner/vendre un canard à moitié en faire accroire à qqn, mystifier qqn

(E) to lead s.o. up the garden path

beau temps pour les canards

temps pluvieux

(E) fine weather for ducks

bouillon de canard (19)

eau

(E) dog's soup, fish-broth

ça glisse comme sur les plumes d'un canard cela ne fait aucune impression, cela laisse indifférent

(E) it's like water off a duck's back

ça ne casse pas trois pattes à un canard (20)

c'est d'une banale médiocrité

(E) it's nothing to write home about canard

1. cf. bique 1 (1881)

2. fausse nouvelle, rumeur (1750)

3. journal (médiocre) (1848)

4. journaliste (1962)

5. traînard (1808)

6. fausse note (1834)

7. morceau de sucre trempé dans le café (1840)

8. spéculum

9. arriviste (1975)

10. client difficile (1977)

11. baiser sur la bouche (1980)

(E) 2. blue duck

3. rag

4. journalist

5. snail

6. goose

7. lump of sugar dipped in coffee

8. speculum

9. careerist, pusher

10. hard to please customer

11. kiss on the mouth

canard boiteux

1. personne inefficace (1900)

2. entreprise peu rentable (20)

(E) 1. inefficient person

2. lame duck (industry)

canard qui bat des ailes et plonge dans l'onde est signe de pluie à la ronde

dicton qui s'explique de soi-même

(E) when a duck claps its wings and dives

into the water rain will soon come canarder

1. cf. brider la bécasse (1860)

2. tirer des coups de feu sur (1847)

donner des canards à qqn (1690)

ne pas tenir ce qu'on a promis

(E) not to keep one's promise

il fait un froid de canard (19)

le temps est très froid

(E) it's beastly cold, it's a brass monkey

weather, it's cold enough to freeze the balls off a brass monkey

il ne faut pas prendre les enfants du bon Dieu pour des canards sauvages (20)

il ne faut pas trop se moquer des gens

(E) don't think you can make fun of people

marcher en canard (20)

marcher les jambes légèrement écartées, les

pieds en dedans, en se dandinant

(E) to gander

mon petit canard!

(cf. ma biche)

mouillé/trempé comme un canard

complètement mouillé (1696)

(E) as wet as a drowned rat

plonger comme un canard

s'esquiver adroitement

(E) to duck out

plonger en canard (1927)

plonger à la verticale

(E) to dive vertically

si le canard crie, c'est signe de pluie

dicton qui s'explique de soi-même

(E) a quacking duck heralds rain

si les canards battent de l'aile dans le ruisseau, il y aura bientôt de l'eau

(cf. ci-dessus)

vilain petit canard (20)

indésirable que l'on tient à l'écart

(E) unpleasant person kept in the

background

\section{CANARI}

changer l'eau de son canari (1894)

(cf. tenir l'âne par la queue)

jamais corbeau n'a fait un canari

(cf. l'AIGLE n'engendre pas la colombe)

\section{CANE}

bec-de-cane

1. cf. basset 2 (1905)

2. deuxième pène d'une serrure (19)

3. poignée de porte (1881)

4. sorte de pince plate (19)

(E) 2. slide-bolt 
3. lever-handle

4. crowbill, crow's bill

caner (1821)

(cf. faire la cane 3)

ce n'est pas le fient de ses canes (1640)

il le tient en piètre estime

(E) he thinks very little of him

chut/motus, la cane pond! (1640)

silence, taisez-vous!

(E) mouse!

être comme une poule qui a couvé des œufs de cane

être déconcerté par un résultat imprévu

(E) to stare like a throttled cat/like a dead

pig/like a stuck pig

faire la cane

1. cf. mettre la table pour les asticots

2. cf. plonger en canard (1928)

3. reculer, se comporter lâchement (16)

(E) to bug out, to chicken out, to turn chicken

il est comme les canes, toujours le bec en l'eau (1640)

c'est un buveur invétéré

(E) he drinks like a fish, he's a booze-hound marcher comme une cane

(cf. marcher en canard)

quand les canes vont aux champs, les premières vont devant (1640)

réponse ironique à qui demande "quand?»

à tout bout de champ

(E) a derisive retort to s.o. who is ever asking "when?"

\section{CANICHE}

avoir une faim caniche (1851)

avoir grand faim

(E) to be as hungry as a bear/horse/wolf, to

feel like one could eat a horse, to have a wolf in one's belly, the stomach-worm gnaws

ça n'est pas fait pour les caniches

c'est fait pour qu'on s'en serve

(E) it's not just as an ornament, it's there to be used

suivre comme un caniche

suivre docilement

(E) to follow about like a $\underline{\operatorname{dog}}$

\section{CARPE}

bâiller comme une carpe (1867)

bâiller sans mesure

(E) to yawn one's head off

c'est le mariage de la carpe et du lapin (1934) ce sont deux choses mal assorties

(E) it's quite a bad match

faire des sauts de carpe (19)

faire des culbutes

(E) to flop about, to somersault

faire des yeux de carpe frite

faire les yeux doux à qqn (1745)

(E) to make lamb's/sheep's eyes at s.o., to have a lovebird look in one's eyes

faire des yeux de carpe pâmée

se pâmer (de plaisir) (1867)

(E) to turn up one's eyes like a dying duck in a thunderstorm

faire la carpe (1864)

(cf. ci-dessus)

faire la carpe pâmée (1835)

feindre de se trouver mal

(E) to stimulate a fit of faintness

faire l'œil de carpe à qqn (1867)

(cf. faire des yeux de carpe frite)

ignorant/niais comme une carpe

(cf. bête comme un âne) (1867)

la carpe saute, de l'eau sans faute

si l'on voit la carpe sauter hors de l'eau, il

faut s'attendre à de la pluie

(E) if the carp jumps out of the water it will soon rain

muet comme une carpe (1612)

absolument silencieux

(E) as dumb, as mute as a fish, as mute as a $\underline{\text { maggot, }}$ as close as a oyster

rester comme une carpe qui perd l'eau (1894)

(cf. être comme une poule qui a couvé des œufs de CANE)

s'embêter/s'ennuyer comme une carpe (1867)

s'ennuyer ferme

(E) to be bored stiff

\section{CASTOR}

castor

1. cf. araignée de trottoir (17)

2. bonneteur (1892)

3. homosexuel, prostitué (1928)

4. homme d'une grande puissance sexuelle (1970)

5. mousse de la marine (20)

(E) 2. card-sharp

3. bitch, chick(en), gay-cat

4. donkey-rigged man

5. ship's boy

demi-castor (1695)

femme de moyenne vertu

(E) woman of loose morals 


\section{CERF}

au cerf la bière, au singulier le barbier (1690) les blessures du cerf sont mortelles, à celles du sanglier peut remédier le chirurgien (E) the wounds from a stag are lethal, those from a boar are for the surgeon's knife

bander comme un cerf (1953)

avoir une forte érection

(E) to have a long pig, to have a rock python corne de cerf!

(cf. ventre de biche!)

courir comme un cerf (19)

courir très vite

(E) to run like a deer/hare, to hare

on connaît le cerf aux abattures

un seul trait suffit pour reconnaître la valeur

d'un homme

(E) by this claw you may know the lion se déguiser en cerf

1. cf. courir comme un cerf (1846)

2. prendre la fuite (1872)

(E) to beetle off, to hare off, to turkey off vin de cerf (1640)

vin qui fait pleurer le buveur

(E) wine that saddens the drinker

\section{CHABOT}

donner un chabot pour avoir un gardon

(cf. donner un œuf pour avoir un bœuf)

\section{CHACAL}

chacal

1. cf. bourrique 5 (1941)

2. zouava (mil.) (1836)

3. âme damnée de qqn (1857)

(E) 2. zouave (mil.)

3. jackal

\section{CHAMEAU}

chameau

1. cf. sale bête 2 (1828)

2. individu odieux; mégère (19)

3. table d'examen gynécologique (1950)

(E) 2. beast, bitch (f), son of a bitch, cat (f), cow (f), dirty dog, hog, louse, mare (f), pig, dirty rat, swine, tick, tyke

3. gynecologist's inspection table

il est plus facile à un chameau de passer par le trou d'une aiguille qu'à un riche d'entrer dans le ciel (13)

formule évangélique qui fustige les riches

(E) it is easier for a camel to go through the eye of a needle than for a rich man to enter into the kingdom of God rejeter le moucheron et avaler le chameau (1842) éviter les petites fautes et s'en permettre de grandes

(E) to strain at a gnat and swallow a camel sobre comme un chameau

d'une grande sobriété

(E) abstemious

vieux chameau (1867)

1. cf. sale bête 1 et 2

2. cf. bique 2

\section{CHAMELLE}

chamelle

1. cf. bourrin 4

2. cf. araignée de trottoir

\section{CHAPON}

avoir les mains faites en chapon rôti

1. avoir des mains déformées par la goutte

(1640)

2. avoir les mains crochues d'un voleur

(E) 1. to have gouty fingers

2. to be light fingered

cage à chapons (1867)

couvent d'hommes

(E) men's convent

ce sont deux chapons de rente, l'un maigre et l'autre gras (1690)

ce sont deux choses tout à fait différentes, c'est tout autre chose

(E) that's a horse of another colour

chapon de Gascogne (1787)

croûton de pain frotté d'ail

(E) crust rubbed with garlic

chapon de huit mois, manger de rois

la chair du jeune chapon est délicieuse

(E) the flesh of a young capon is delicious chapon de Normandie (1640)

croûton de pain dans la soupe

(E) crust in soup

chapon de Limousin

châtaigne

(E) chestnut

jamais géline n'aima chapon (1568)

les femmes aiment les hommes virils qui chapon mange, chapon lui vient (1611)

les biens viennent à ceux qui en ont déjà

(E) he that hath, to him shall be given qui chapon mange, perdrix lui vient (1640)

(cf. ci-dessus)

\section{CHARANÇON}

charançons (1960)

gonocoques (propagateurs de la blennorragie)

(E) gonococci 


\section{CHAT}

à bon chat, bon rat (1585)

à bon attaquant, bon défenseur; les choses

doivent être proportionnées

(E) two can play at that game

absent le chat, les souris dansent (1575)

quand l'autorité est absente, le désordre règne

(E) when the cat's away the mice will play

acheter chat en poche (14)

faire un marché de dupe; être imprudent

(E) to buy a dog in blanket/a pig in a poke

acheter un chat pour un lièvre

être trompé sur la marchandise

(E) to be cheated on the goods

aller comme un chat maigre (16)

courir

(E) to run

aller du pied comme un chat maigre (1640)

marcher vite

(E) to walk at a quick pace

appeler un chat un chat (17)

dire les choses franchement

(E) to talk turkey

arriver dès les chats

(cf. au chant de l'alouette)

attacher la sonnette au cou du chat (1640)

se lancer dans une entreprise dangereuse

(E) to bell the cat

avoir avalé un chat par la queue

être enroué

(E) to have a frog in one's throat

avoir d'autres chats à fouetter (18)

avoir à faire des choses plus urgentes, plus

importantes

(E) to have other fish to fry

avoir joué avec les chats

être porteur d'égratignures (au visage)

(E) they that jest with cats must count on scratches

avoir l'air d'un chat qui chie dans la braise avoir l'air ridicule

(E) to look like a billy-goat in stays

avoir un chat dans la gorge (1835)

(cf. avoir avalé un chat par la queue)

avoir un chat à la poêle, l'autre au chat (16)

être vigilant, surveiller

(E) to keep one's eyes skinnes

bailler le chat par les pattes (17)

présenter une chose par son côté le plus

difficile

(E) to present sthg by its bad side

barbes de chat aux nuages annoncent de vent tapage

des nuages effilochés sont signe de grand vent

(E) fine-drawn clouds herald wind bien sait le chat quelle barbe il lèche (13)

le rusé est toujours prudent

(E) the cat knows whose lips she licks

bouffer un chat (1928)

faire un cunnlingus

(E) to eat a kipper feast, to have a tuna taco (US)

bouillie pour les chats (1789)

chose mal réussie, travail gâché

(E) sthg just fit pour the cat

c'est cacher une souris dans l'oreille d'un chat

(17)

c'est mettre une chose à la disposition de qui la convoite

(E) it's putting sthg in the hands of s.o. who lusted for it

c'est ici que les chats se peignent! (1894)

voilà où gît la difficulté

(E) that's the fly in the ointment!, thats where the hen scratches!

c'est le chat! (1867)

répartie à qqn qui s'écrie "c'est pas moi!"

(E) jocular retort to s.o. who exclaims "it's not me!"

c'est le gros rat qui a mangé le chat des carmes (1640)

c'est une affaire sans importance

(E) it's a matter of no importance

c'est le nid d'une souris/c'est un nid de souris

dans l'oreille d'un chat (1690)

c'est une chose impossible

(E) it's a bold/wily mouse that breeds/nestles in the cat's ear

ce n'est pas fait pour les chats

(cf. ça n'est pas fait pour les caniches) chat

1. cf. angora (1862)

2. toison pubienne de la femme (16)

3. geôlier (1828)

4. greffier de tribunal (1953)

(E) 2. bearskin, beaver, cat-skin

3. yard-bull

4. clerk of the court

chat à dix doigts (1640)

être humain

(E) humain being

chat à neuf queues (1845)

martinet

(E) cat-o'-nine-tails

chat échaudé craint l'eau froide (13)

on redoute même l'apparence de ce qui a

déjà nui

(E) a scalded cat fears cold water, the

escaped mouse ever feels the taste of the bait 
chat emmitouflé ne prend (point de) souris (1597)

ne peut faire qqch habilement avec des gants aux mains

(E) a cat in gloves catches no mice, a mittened cat never was a good hunter, muffled cats are not good mousers

chat et chaton chassent le raton

(cf. bon chien chasse de race) chat fourré

1. juge (1532)

2. médecin (1640)

(E) 1. beagle (US)

2. physician

chat qui est accoutumé à/de prendre des souris ne peut s'en tenir (1640)

le méchant se défait difficilement de ses mauvaises habitudes

(E) the fox may grow grey but never good chats passant pattes sur l'oreille, s'il vient beau temps serait merveille

si le chat passe la patte sur l'oreille en faisant sa toilette il faut s'attendre à la pluie

(E) if the cat reaches over its ear with its paw rain is to be expected courir comme un chat maigre

(cf. courir comme un cerf)

dès que les chats sont chaussés (1690)

(cf. au chant de l'alouette)

donner sa langue au chat (19)

1. renoncer à deviner qqch

2. avouer sa totale ignorance

(E) 1. to give up guessing

2. to confess one's ignorance

du pipi de chat (20)

(cf. c'est de la pisse d'âne)

écrire comme un chat (1853)

écrire de manière illisible

(E) to make hen scratches (US)

elle en donne aux chiens et aux chats

c'est une marie couche-toi-là (1640)

(E) she's got a colt's tooth

emporter le chat

1. s'en aller sans payer ou sans prendre congé

(1640)

2. se mêler d'une chose que l'on ne connaît

pas et en recevoir injure (1867)

(E) 1. to shoot the crow

2. to meddle in sthg one does not know and get abused for it

entendre bien chat sans qu'on dise minou (1640)

comprendre à demi-mot

(E) to catch on at once être amis comme (le) chien et (le) chat (1531)

être en désaccord, en dispute

(E) to agree/live/quarrel like cat and dog, to lead a cat-and-dog life

être comme chat sur braise

éprouver une vive impatience,

être dans l'agitation

(E) to be like a cat on hot bricks/on a hot roof (US), to sit on a bag of fleas, to be like a fly in a glue-pot/tar-pot), to be like a hen on a hot griddle, to be as quiet as a wasp in one's nose

éveillé comme un chat qu'on fouette (17) rusé, roublard

(E) as sly as an old fox, foxy

faire de la bouillie pour les chats (1867) travailler sans profit pour soi ni personne

(E) to flog a dead horse

fouetteux de chats (1867) personne sans énergie, sans force morale (E) spineless character

fréquenter le chien et le chat fréquenter tout le monde

(E) to mix with everyone

guetter qqn comme le chat (fait de) la souris (17) épier qqn

(E) to watch s.o. like a cat watching a mouse

il a été au trépassement d'un chat (1640)

il a trop bu, il voit double

(E) he's been drinking, he sees double

il n'y a pas de quoi étouffer un chat

(cf. ça ne casse pas trois pattes à un canard)

il n'y a pas de quoi fouetter un chat

ce n'est rien de grave, c'est une vétille

(E) it's a mere hen cackle

il n'y a pas la queue d'un chat (1867)/pas un chat (1778)

il n'y a absolument personne

(E) there's not a living soul

il n'y a pas de si petit chat qui n'égratigne (17)

il n'y a pas de petit ennemi

(E) be thine enemy an ant, see in him an

elephant, even a worm will turn, tread on a worm and it will turn

il ne faut pas faire passer tous les chats pour des sorciers (1842)

il ne faut pas conclure du particulier au général

(E) do not go from the particular to the general

il ne faut pas jouer avec les chats (1640)

il faut éviter les familiarités avec qui peut vous nuire

(E) they that jest with cats must count on scratches 
il ne faut pas réveiller le chat qui dort (1568)

il ne faut pas relancer une affaire fâcheuse, une querelle assoupie

(E) let sleeping dogs lie, let that fly stick to the wall, wake not a sleeping lion

inutile de gronder le chat quand le fromage est mangé

les mesures tardives sont inutiles

(E) it's not use bolting the stable-door after the horse is gone, it's too late to shut the stable-door when the steed is stolen

jeter le chat aux jambes de qqn (1640)

rejeter la faute sur autrui

(E) to lay the blame at s.o.'s door

jouer au chat et à la souris avec qqn (19)

s'amuser cruellement de qqn que l'on tient en son pouvoir

(E) to play cat and mouse with s.o.

la nuit tous les chats sont gris (17)

dans l'obscurité il est facile de confondre les personnes et les choses

(E) all cats are grey in the dark, when

candles are away all cats are grey

là où le chat n'est, la souris se révèle (13)

(cf. absent le CHAT les souris dansent)

laisser le chat aller au fromage (16)

coïter, du point de vue de la femme

(E) to take in beef, to give mutton for beef, to home the pigeon, to feed one's pussy, to tame a serpent, to grease the pants' worm

le chien commande au chat et le chat à sa queue (1640)

on essaye toujours de faire faire son travail par autrui

(E) one always tries to order s.o. else to do one's job

le singe tire les marrons du feu avec la patte du chat (1576)

l'homme habile fait prendre les risques par autrui

(E) to take the chestnuts out of the fire with the cat's paw

maigre comme un chat de gouttière

(cf. maigre comme un chien fou)

mon chat a le nez cassé (1864)

j'ai mes règles

(E) I'm riding the white horse

(US) Mickey Mouse is kaput

mon petit chat! (1672)

(cf. ma biche!)

musique de chats

miaulements

(E) cats' chorus, suffering cats

n'éveillez pas le chat qui dort (1531)

(cf. il ne faut pas réveiller le chat qui dort) occasion trouve qui son chat bat (1495)

quand on en veut à qqn on l'accuse

faussement, on le déprécie

(E) a stick is quickly found to beat a dog

with, give a dog a bad name and hang him,

he that would hang his dog gives out first

that he is mad

ouvrir les yeux comme un chat qui coud dans une gouttière (1640)

(cf. être comme une poule qui a couvé des oufs de CANE)

passer sur qqch comme chat sur braise (16)

éviter de s'appesantir sur qqch

(E) to glide over sthg without insisting

payer en chats et en rats (1690)

payer par petites sommes ou en objets de peu de valeur

(E) to pay in small amounts or in goods of little value

penaud comme un chat qu'on châtie (1640)

embarrasé, interdit, honteux

(E) looking sheepish

prendre un plat de chat (1861)

(cf. bouffer un chat)

présenter le chat par les pattes (1690)

(cf. bailler le chat par les pattes)

propre comme une écuelle à chat (1690)

sale et mal mis

(E) filthy and sloven

quand le chat n'est pas là, les souris dansent (19)

(cf. absent le CHAT, les souris dansent)

quand le chat se débarbouille, bientôt le temps se brouille

(cf. chats passant pattes sur l'oreille, s'il vient

beau temps serait merveille)

quand le chat se passe la patte sur la tête,

bientôt il y aura tempête

(cf. ci-dessus)

qui naquit chat court après la souris (1842)

(cf. demande-t-on à des béliers qu'ils n'aient pas de cornes)

qui ne nourrit pas le chat nourrit le rat

en voulant s'épargner une dépense on risque un dommage plus grand

(E) he that spares the bad injures the good qui perd un chien et retrouve un chat, c'est toujours une bête à quatre pieds

(cf. bête)

retomber sur ses pattes comme un chat

arriver toujours à se tirer d'affaire sans

dommage

(E) to always fall on one's feet

s'accorder/s'entendre comme chien et chat (1640)

(cf. être amis comme chien et chat) 
se servir de la patte du chat pour tirer les marrons du feu

(cf. le singe tire les marrons du feu avec la patte du CHAT)

si les chats gardent les chèvres, qui attrapera les souris?

à chacun son métier

(E) every man to his trade

tant dort le chat qu'il se réveille

l'impunité rend téméraire

(E) impunity leads to foolhardiness

tous les chats sont gris la nuit (19)

(cf. la nuit tous les chats sont gris)

un vieux chat aime les jeunes souris (1456)

le vieillard amoureux se tourne vers les

jeunettes

(E) old men are attracted by young girls

vivre comme chien et chat (17)

(cf. être amis comme chien et chat)

vous avez un vouloir, notre chat en a deux (1640)

répartie à qqn qui prétend absolument

obtenir qqch

(E) retort to s.o. who insists on absolutely getting sthg

\section{CHATON}

chat et chaton chassent le raton

(cf. bon chien chasse de race)

chatons

inflorescences

(E) catkins, lamb's tails

\section{CHATTE}

à laide chatte, beaux minous

une femme laide attire souvent les beaux jeunes gens

(E) an ugly woman is often courted by

handsome young men

amoureuse comme une chatte

très amoureuse

(E) of an amorous disposition

aux vilains matous de belles chattes (1912)

les jolies femmes d'éprennent souvent

d'hommes laids

(E) pretty woman often fall for ugly men

avoir la chatte à l'agonie (20)

être excitée sexuellement

(E) to be aroused sexually (f) chatte

1. cf. angora (1930)

2. cf. bourrique 5 (1836)

3. cf. castor 3 (1883)

4. femme débauchée, allumeuse (1883)

(E) débauched woman, vamp ma petite chatte!

(cf. ma biche)

se mettre la chatte en robe à queue (1953)

(cf. avoir la chatte à l'agonie)

une chatte n'y retrouverait pas ses jeunes/ses petits (20)

c'est un grand désordre

(E) it's a regular beargarden, it's a dog's/pig's

breakfast, it's a pretty kettle of fish

\section{CHAUVE-SOURIS}

chauves-souris volant en grand nombre

annoncent le beau temps dans la nuit sombre

dicton qui s'explique de soi-même

(E) bats in swarm are a sign of fair weather

\section{CHENILLE}

c'est une chenille!

c'est un importun

(E) he's a regular pest!

chenille (17)

1. exploiteur (du peuple)

2. importun dont on ne peut se défaire

(E) 1. caterpillar (of society)

2. barnacle, leech, limpet

grippe, chenille! (1640)

prends! attrape!

(E) take that

méchante chenille (1690)

(cf. mauvaise bête)

ôtez les ailes à un papillon, c'est une chenille (18)

le fat n'existe que par sa vanité

(E) a vain person exists only by his conceit

\section{CHEVAL}

à bon/grand cheval, bon/grand gué (15)

il faut s'adapter aux circonstances

(E) one must adapt to circumstances

à cheval donné, on ne regarde point la bouche/

la bride/la dent (1690)

on ne doit pas critiquer les choses que l'on reçoit

(E) look not a gift horse in the mouth, never look a gift horse in the mouth

à cheval hargneux il faut une écurie à part (1786)

il faut tenir éloignés les gens querelleurs

(E) quarrelsome people must be held apart

à cheval maigre vont les mouches (1576)

on persécute surtout les faibles

(E) a lean dog gets nothing but fleas

à jeune cheval, vieux cavalier (16)

il faut un cavalier expérimenté pour monter un jeune cheval 
(E) a young trooper should have an old horse à jeune homme vieux cheval, à jeune cheval vieil homme (1852)

(cf. ci-dessus)

à méchant cheval, bon éperon (16)

(cf. à dur âne, dur aiguillon)

à pied, à cheval et en voiture (19)

de toutes les manières, à fond

(E) by all means, thoroughly

après bon vin, bon cheval (1690)

on fait plus de diligence quand on est repu

(E) one shows greater diligence when

satiated

avoir mangé du cheval

être plein d'énergie, de vitalité

(E) to look as if one had eaten live birds

bon cheval de trompette (1842)

individu qui ne s'effraie pas facilement

(E) a man not easily frightened

brider son cheval par la queue (1640)

(cf. brider son âne par la queue)

c'est en vain qu'on mène à l'abreuvoir un cheval qui n'a pas soif

(cf. on ne saurait faire boire un âne qui n'a pas soif)

c'est la mort du petit cheval! (20)

c'est la fin de tout

(E) that's the end of the line!

c'est pas un mauvais cheval (1931)

c'est un type sympathique malgré son

comportement bourru

(E) he's not a bad sort

c'est un bon cheval de trompette (1842)

il n'est pas vite effrayé

(E) he lives too close to the wood to be

frightened by an owl

ce n'est pas le cheval qui tire mais l'avoine

il faut être stimulé pour produire

(E) it is by the head that the cow gives milk,

money makes the mare to go

ce ne sont pas les chevaux qui tirent le plus fort qui mangent l'avoine (17)

(cf. les chevaux courent les bénéfices et les

ÂNES les attrapent)

cela ne se trouve pas sous le pas/le sabot d'un cheval (1640)

c'est une chose rare

(E) it does not grow on every hedge

celui qui ne s'aventure n'a ni cheval ni voiture

qui ne risque rien n'a rien

(E) nothing venture, nothing win

changer/troquer son cheval borgne pour un

aveugle (1616)

(cf. quitter un bœuf pour prendre un œuf) chercher à cheval et en voiture (1640)

chercher avec grand soin

(E) to search thoroughly

cheval

1. cf. bourrin 5 (1975)

2. forte carte au baccara (1878)

3. bubon à l'aine (1878)

4. femme hommasse (grand cheval)

(E) 2. master card at baccara

3. blue boar, Winchester goose, French pig, Winchester pigeon

4. bull bitch, horse-godmother

cheval aux quatre pieds blancs (1640)

grand prometteur

(E) person quick to promise

cheval d'avoine, cheval de peine (1640)

le cheval nourri à l'avoine travaille bien

(E) a horse fed on oats works hard

cheval de bagage (1640)

(cf. âne 1 et gros bouf)

cheval de bât (1690)

(cf. animal 5)

cheval de bataille (1690)

sujet préféré, argument favori

(E) stock answer or argument

cheval de carrosse (1690)

(cf. âne 2)

cheval de charrue (1690)

(cf. âne 2 et animal 5)

cheval de foin, cheval de rien (1640)

un cheval nourri au foin est sans vigueur

(E) a horse fed on hay has no strength

cheval de paille, cheval de bataille (1640)

le cheval nourri à la paille travaille mieux

(E) a horse fed on straw works better

cheval de retour (1828)

évadé repris, récidiviste

(E) old jail-bird

cheval échappé (17)

débauché, libertin

(E) gay $\underline{\operatorname{dog}}$, wolf

cheval fait et femme à faire (1640)

il faut prendre un cheval tout dressé et une

femme à former à son goût

(E) choose a horse made and a wife to make cheval qui piaffe n'avance guère

l'agitation est stérile

(E) agitation is unproductive

compte plutôt sur ton âne que sur le cheval de ton voisin

(cf. âne)

coucher/pioncer avec le cheval (1844)

dormir seul

(E) to sleep alone 
culotte de cheval (20)

cuisses féminines envahies par la cellulite

(E) woman's thighs invaded by cellulitis

dîner/manger avec les chevaux de bois (1949)

se passer de manger

(E) to go dinnerless

être à cheval

1. être dans l'aisance (1640)

2. avoir purgé la moitié de sa peine de prison

(1880)

(E) 1. to be in the bucks (US)

2. to be halfway through a prison sentence

être à cheval sur qqch (1836)

se montrer ferme/strict sur qqch

(E) to be a strickler for sthg

être à cheval sur un torchon (19)

(cf. mon chat a le nez cassé)

être mal à cheval

1. être mal à l'aise (1640)

2. être proche de la ruine (1690)

(E) 1. to feel uneasy

2. to be going to the dogs

être un cheval à l'ouvrage

(cf. être un bœuf pour le travail)

faire son cheval de corbillard (1883)

faire le malin, poser

(E) to show off, to swank

fermer l'écurie quand les chevaux sont dehors prendre des précautions alors qu'il est trop tard

(E) to close the stable-door when the steed is stolen

fièvre de cheval (1798)

forte fièvre

(E) raging fever

gros cheval (1690)

(cf. âne 2)

histoires à la graisse de chevaux de bois (1910) excuses ou prétextes grossiers

(E) a lot of bull(shit)

il est comme les chevaux de trompette, il boit à tout gué (1640)

il boit et mange partout et en toute occasion

(E) he eats and drinks everywhere and in any circumstances

il est trop tard de fermer l'écurie quand le cheval s'est sauvé (13)

(cf. inutile de gronder le chat quand le fromage est mangé)

il fait bon aller à pied quand on mène son cheval par la bride (1640)

la contrainte est légère quand on a le moyen d'y mettre fin
(E) constraint is light when one has the means of putting an end to it

il fait toujours bon tenir son cheval par la bride il est bon de veiller assidûment à ses intérêts

(E) one must attend to one's interests unremittingly

il faut mettre une barre entre eux deux comme aux méchants chevaux (1640)

(cf. à cheval hargneux il faut une écurie à part)

il n'est si bon cheval qui ne bronche (1640)

1. cf. à bon pêcheur échappe l'anguille

2. même l'homme le plus sage peut commettre un écart

(E) even the steadiest man may have his fling

il n'est si bon cheval qui n'en devienne rosse (1690)

1. l'homme le plus robuste devient caduc avec l'âge

2. si l'on accable qqn d'un trop grand travail il peut y succomber

(E) 1. the most sturdy man is broken down by old age

2. too heavy a burden of work may be fatal

il se tient mieux à table qu'à cheval (1640) c'est un goinfre

(E) he's a greedy pig

il vaut mieux être cheval que charette (1821) mieux vaut commander qu'obéir

(E) better order than be ordered

j'en parlerai à mon cheval!

je me moque de ce que tu me dis, cela ne m'intéresse pas

(E) I don't care three skips of a louse about what you say!

jamais bon cheval ne devient rosse (1548)

les bonnes qualités ne se perdent jamais entièrement

(E) good qualities are never entirely lost jamais coup de pied de jument ne fit mal à un cheval (1835)

un homme ne s'offense pas injures d'une

femme

(E) a man does not take offence at the

wrongs of a woman

jamais mauvais cheval ni méchant homme n'amenda pour aller à Rome

(cf. qui bête va à Rome tel en retourne) je lui ferai voir que son cheval n'est qu'une bête! (1690)

(cf. bête) 
l'âge n'est (fait) que pour les chevaux (1690) on n'apprécie à l'âge que les chevaux, non les gens

(E) age is a standard for horses, not for men l'avoine fait le cheval

(cf. ce n'est pas le cheval qui tire, mais l'avoine)

l'écurie use plus le cheval que la course l'inaction/l'oisiveté émousse les énergies

(E) idleness blunts energy

l'œil du maitre engraisse le cheval (1568)

1. cf. il fait bon toujours tenir son cheval par la bride

2. tout va mieux quand le maître est présent

(E) the master's eye makes the horse fat

le cheval a quatre pattes et pourtant il bronche (15)

(cf. il n'est si bon cheval qui ne bronche)

le cheval qui traîne son lien n'est pas échappé (1568)

n'est jamais libre qui ne rompt ses attaches

(E) the horse that draws his halter is not quite escaped

les bons chevaux s'échauffent en mangeant

(1616)

manger échauffe le tempérament

(E) eating fires temperaments

les chevaux courent les bénéfices et les ânes les attrapent (1842)

(cf. âne)

les maladies viennent à cheval et s'en retournent à pied

les maladies disparaissent moins vite qu'elles ne surviennent

(E) agues come on horseback but go away on foot

méchant poulain peut devenir bon cheval

on se corrige avec l'âge

(E) a ragged colt may make a good horse

médecine de cheval (17)

remède énergique

(E) horse medicine

mémoire de cheval

excellente mémoire

(E) excellent memory

mener son cheval par la bride (1640)

être assuré de sa réussite

(E) to be assured of one's success

mettre qqn à cheval (1640)

faciliter les débuts de qqn

(E) to give s.o. a leg up

miser sur le mauvais cheval (20)

commettre une erreur d'appréciation

(E) to set the saddle on the wrong horse monter à cheval comme une paire de pincettes/ comme une paire de tenailles sur le cul d'un chien (1894)

être mauvais cavalier

(E) that must have been a butcher's horse by his carrying a calf so well, to sit like a

monkey on a gridiron, who put that monkey on horseback without tying its tail?, to sit like a toad on a chopping-block

monter sur ses grands chevaux

1. cf. piquer un bœuf (1585)

2. le prendre de haut (1842)

(E) to get on one's high horse, to ride the high horse

mors doré ne rend pas le cheval meilleur une mise recherchée ne change pas le cœur de l'homme

(E) a richer attire does not make a better man

n'attelle pas ensemble l'âne et le cheval (14)

(cf. âne)

n'avoir ni cheval ni mule

être sans ressources

(E) to be full of money as a toad is of feathers

on achète les bons chevaux à l'écurie (B) une jeune fille n'a pas à courir les bals pour trouver un mari

(E) a well-bred girl need not be a ball fiend to find a husband

on avance mieux avec un cheval emprunté

qu'avec le sien propre

l'homme a tendance à épargner son bien

(E) a hired horse never tires

on frappe toujours sur le cheval qui tire (1640) on en demande toujours plus au plus occupé

(E) all lay load on the willing horse

parler à cheval (19)

parler mal, de façon incorrecte

(E) to murder the language

plus tire nature que cent chevaux

l'attrait de l'éternel féminin est puissant

(E) nature draws more than ten oxen, one

hair of a woman can draw more than a team of oxen

pousser son cheval (1640)

poursuivre son dessein

(E) to pursue one's puropose

prendre un cheval par les crins (17)

entreprendre de grandes choses, avoir de

l'ambition

(E) to have great projects, to be ambitious

quand le foin manque au râtelier, les chevaux se battent (1860)

(cf. quand il n'y a plus de foin au râtelier, les ânes se battent) 
queue de cheval (20)

type de coiffure féminine

(E) horse tail

qui veut un cheval sans défaut doit aller à pied (13)

la perfection n'existe pas

(E) perfection is not of this world

selle à tous les chevaux (17)

discours passe-partout, qui sert en toute occasion

(E) general-purpose speech

si le cheval se connaissait cheval, il voudrait être homme (1557)

(cf. il vaut mieux être cheval que charrette) tous ne sont pas chevaliers qui à cheval montent (13)

(cf. les grands bœufs ne font pas les grands labours)

tout cheval a besoin d'éperon (17)

(cf. âne piqué à trotter est incité)

traîner le cheval mort (1867)

exécuter un travail payé d'avance

(E) to draw/pull a dead horse, to work for a/ the dead horse

travail de cheval (1690)

travail épuisant

(E) hard work

travailler comme un cheval (18)

(cf. travailler comme une bête)

trop piquer le cheval le fait rétif (1585)

les remontrances incessantes provoquent une réaction opposée à celle souhaitée

(E) do not spur a free horse

troquer son cheval borgne pour un aveugle (1616)

(cf. quitter un bœuf pour prendre un œuf) vendre son cheval pour avoir l'avoine (1640) se défaire du principal pour l'accessoire

(E) to sell the nore valuable to get less

\section{CHÈVRE}

à la chandelle, la chèvre semble demoiselle (1576) dans la pénombre, la fille légère ne se distingue pas de la fille sérieuse

(E) in the dark it is difficult to tell a fast girl from a serious one

avoir la chèvre (1867)

(cf. avoir son bœuf)

barbe de chèvre (1640)

une grande barbe n'est pas signe de grand savoir

(E) if the beard were all, the goat might preach chèvre (1930)

fille ou femme légère, portée sur le sexe

(E) hot mutton

devenir chèvre (1675)

(cf. piquer un bœuf)

faire devenir chèvre (1932)

(cf. faire tourner en bourrique)

gober la chèvre (1867)

1. cf. être le bœuf

2. essuyer la mauvaise humeur de qqn

(E) to endure s.o.'s grumpiness

il aimerait une chèvre coiffée (1842)

il s'éprend de toutes les femmes

(E) he runs after every petticoat

la barbe lui croît avant l'entendement comme aux chèvres de Brie (1640)

il a plus de barbe que d'intelligence

(E) his beard outgrows his judgment

la chèvre a pris le loup (1752)

le plus faible l'a emporté sur le mal intentionné à son égard

(E) it's a case of the biter bit

la chèvre a sauté en la vigne, aussi y sautera la

fille (13)

telle mère, telle fille

(E) like mother, like daughter

ménager la chèvre et le chou (1672)

se garder de prendre parti

(E) to hold with the hare and run with the

hounds, to run with the hare and hunt with the hounds

on n'a jamais vu chèvre morte de faim (1842)

quand on n'est pas difficile, on survit

(E) don't be too particular about food if you

will survive

où la chèvre est attachée/liée, il faut qu'elle

broute (1598)

il faut s'accomoder de sa situation, de son

milieu

(E) the goat must browse where she is tied, where she is tied, where shall the ox go but she must labour, a man must plough with such oxen as he has

prendre la chèvre

1. cf. piquer un bouf (16)

2. cf. être le bouf (17)

3. cf. gober la chèvre (17)

rendre chèvre

(cf. faire devenir chèvre)

reprenons notre chèvre à la barbe (1640)

revenons à notre propos

(E) to return to one's muttons

ses paroles s'entretiennent comme crottes de chèvre (1640) 
il parle indistinctement

(E) he speaks like a mouse in cheese si les chats gardent les chèvres, qui attrapera les souris?

(cf. chat)

son discours se suit comme crottes de chèvre (1690)

ses propos son incohérents

(E) you cannot make head or tail of his speech

vin à faire danser les chèvres (17)

vin très aigre

(E) very sour wine

\section{CHEVREUIL}

chevreuil

1. cf. araignée de trottoir (1919)

2. cf. bourrique 5 (f) (1925)

3. homme peu fiable, poltron (1912)

4. paysan

(E) 3. fraidy $\underline{\text { cat }}$

4. peasant

\section{CHIEN}

à bon chien il n'arrive jamais bon os (1640) (cf. les chevaux courent les bénéfices et les ÂNES les attrapent)

à chair de loup, dent de chien (17) les moyens doivent être appropriés à l'objectif (E) the means must be adapted to the object à méchant chien, court lien

il ne faut pas laisser trop de liberté aux méchants

(E) the wicked must not be allowed too much freedom

à toute heure chien pisse et femme pleure certains comportements sont inévitables

(E) some behaviours are inevitable

agir comme un chien fouetté

faire qqch de mauvaise grâce

(E) to do sthg ungraciously

aller à nid de chien (1690)

tourner à rien, échouer

(E) to go to dogs (US), to go to pigs and whistles

appétit de hien (1640)

grand appétit

(E) wolfish appetite

autant vaut être mordu d'un chien que d'une chienne (1640)

inutile de choisir entre deux choses à

craindre également

(E) it's a toss-up which is worse of two evils avec les $\underline{\text { chiens }}$ on ne gagne que des puces (1640) fréquenter la mauvaise compagnie laisse des traces

(E) he who lies down with dogs will rise with fleas, sue a beggar and get a louse avoir d'autres chiens à fouetter (1828)

(cf. avoir d'autres chats à fouetter)

avoir du chien (1866)

être séduisante, provocante

(E) to have charm, sex-appeal

avoir du chien dans le ventre

1. être courageux, hardi (1867)

2. être de force à tout supporter (1872)

(E) 1. to be one of the bulldog breed

2. to have the fortitude of enduring everything

avoir du crédit comme un chien à la boucherie (18)

ne jouir d'aucun crédit

(E) to have no credit at all

avoir l'air d'un chien battu

avoir l'air triste, défait

(E) to look as melancholy as a cat/as a sick monkey

avoir la jambe tout d'une venue comme la jambe d'un chien (17)

avoir des jambes très maigres

(E) to have bird-legs/canary legs/cat-sticks avoir un chien d'esprit (1640)

1. cf. avoir une mémoire de cheval

2. avoir l'esprit inventif

(E) to have an inventive mind

avoir un chien pour qqn (19)

être épris(e) de qqn

(E) to be apeshit about s.o. (US), to be batty over s.o., to be Cooper's duck with s.o.

avoir un mal de chien à...

aprouver beaucoup de difficultés

(E) to have a devil of a job to...

avoir une veine de chien courant

être très chanceux

(E) to be a lucky dog

barbe de lièvre, qui n'ose sortir de peur des

chiens (1640)

barbe naissante

(E) incipient beard

battre/traiter en chien courtaud (17)

frapper violemment

(E) to give lamb and salad

battre le chien devant le lion (1640)

corriger qqn en présence d'autrui pour

inspirer la crainte

(E) to chastise s.o. in the presence of others to inspire fear 
bon chien chasse de race (1557)

on hérite généralement des qualités de sa famille

(E) cat will after kind, that that comes of a cat will catch mice

bouillon de chien (19)

pluie

(E) rain

c'est le chien du fourbisseur qui l'a mordu (1640)

il a reçu un coup d'épée

(E) he's got a sword wound

c'est le chien du jardinier, qui ne mange pas de choux et n'en laisse pas manger (17)

il n'aime pas voir autrui accepter ce qu'il dédaigne

(E) he is a dog in the manger

c'est saint Roch et son chien

ils sont inséparables

(E) they are finger and thumb

c'est un beau chien s'il voulait mordre

il n'a pour lui que de fausses apparences

(E) his bark is worse than his bite

c'est un chien qui aboie à la lune

il s'attaque inutilement à plus fort que lui

(E) he needlessly attacks one who is more than a match for him

c'est une charrue à chiens

c'est une association inefficace

(E) it is an unproductive partnership

ça n'est pas fait pour les chiens (1789)

(cf. ça n'est pas fait pour les caniches)

ça ne vaut pas les quatre fers d'un chien

(cf. c'est de la pisse d'âne)

caresse de chien donne des puces! (19)

formule de refus des compliments d'un

flateur dont on se méfie

(E) a phrase to refuse compliments from a sycophant one distrusts

ce n'est pas à vous que le chien en a, mais à

votre pain

le flatteur est souvent intéressé

(E) dogs wag their tails not so much to you as your bread

cela n'est pas tant chien! (1690)

ce n'est pas si mal, si mauvais

(E) it isn't half bad!

chien

1. alcool (de mauvaise qualité) (1825)

2. agent de la police secrète (1828)

3. béguin (19)

4. charme, séduction

5. travail payé d'avance (1867)
(E) 1. bug-juice, catgut, dog juice, hogwash, wild mare's milk, white mule, nanny-goat sweat, panther juice, blind pig, rat, snake juice, tiger's milk
2. sleuthhound
3. infatuation
4. charm, sex-appeal
5. dead horse

chien (adj)

avare

(E) hoggish, piggish

chien au grand collier (1640)

homme important, influent, notable

(E) big bug, big dog with a brass collar

chien-braye (1640)

couard, poltron

(E) fraidy cat, chicken, chicken-hearted

fellow, cow-baby, cow-heart, hen-hearted

fellow, rabbit

chien couchant

flagorneur, homme obséquieux

(E) toad-eater, toady

chien du bord

officier en second (Mar.)

(E) second officer

chien de caserne (1901) / du quartier (1881) /

du régiment (1861)

adjudant

(E) warrant officer

chien de cour (1783)

maître d'études d'un collège

(E) prep master in a college

chien du commissaire (1848)

secrétaire du commissariat

(E) police-station clerk

chien en cuisine son pair n'y désire

on n'aime pas partager une bonne affaire

(E) while the dog gnwas the bone,

companions would be none

chien enragé (1690)

(cf. bouledogue 2)

chien hargneux (17)

individu querelleur

(E) wrangler

chien hargneux a toujours l'oreille déchirée (1678)

les gens querelleurs ont toujours quelque blessure

(E) quarelling dogs come halting home chien mort ne mord plus

(cf. morte la bête, morte le venin)

chien noyé (1872)

(cf. canard 6) 
chiens perdus sans collier (20)

enfants abandonnés, orphelins

(E) waifs and strays, orphans

chien qui aboie ne mord pas (13)

les gens qui crient le plus ne sont pas le plus à craindre

(E) barking dogs do not bite, $\underline{\text { dogs }}$ bark

before they bite, dogs that bark at a distance never bite

chien vert (1880)

valet de pique

(E) knave of spades

coiffure à la chien (1874)

frange sur le front

(E) fringe hairdress

comme les chiens pour mordre le monde (1894)

méchamment

(E) spitefully, wickedly

comme un chien dans un jeu de quilles (1690)

mal à propos, mal venu

(E) like a dog on a putting-green, like a

skunk in a chicken-house

comme un chien qui sort de l'eau

(cf. mouillé comme un canard)

comme un os dans la gueule d'un chien (1640)

à propos, opportunément

(E) apppropriately, timely

coup de chien (1867)

1. procédé déloyal, traitrise

2. dispute soudaine et violente

3. grain, bourrasque

(E) 1. dog-trick, lousy trick

2. swine-up

3. squall

couper la queue à son chien

commettre quelque excentricité pour faire parler de soi

(E) to behave eccentrically to draw attention to oneself

de chien (1872)

locution d'intensification

(E) a beast of, a whale of

deux chiens à un os ne s'accordent (1640)

deux personnes qui prétendent à une même

chose ne peuvent s'entendre

(E) two dogs and a bone never agree in one, two sparrows on one ear of corn make an ill agreement

dîner de chien (17)

nourriture immangeable

(E) dog's vomit, pigwash

discours à tuer chien (1640)

paroles ridicules

(E) stupid talk dormir en chien (16)

dormir au soleil pendant la chaleur

(E) to bask in the sun

écorcher son chien pour en avoir la peau

sacrifier une chose de valeur pour une autre qui n'en aucune

(E) you can have no more of a cat than the skin

elle en donne aux chiens et aux chats (1640)

(cf. chat)

entre chien et loup (13)

à la tombée du jour

(E) in the cock-shut light/time

entrez, nos chiens sont liés! (17)

il n'y a plus de danger

(E) danger is over now!

être amis comme chien et chat (16)

(cf. chat)

être chien avec qqn (1847)

traiter qqn durement

(E) to be a perfect pig/swine to s.o., to treat s.o. like a toad under the harrow

être coiffée à la chien (1874)

porter une frange sur le front

(E) to wear a straight fringe

être comme le chien du jardinier (1752)

(cf. c'est le chien du jardinier, qui n'aime pas les choux et n'en laisse pas manger)

être comme un chien à l'attache

être dans l'impuissance d'agir

(E) to be tied hand and foot

être d'une humeur de chien (19)

être de très mauvaise humeur

(E) to be as surly as a bear, to be like a bear with a sore head, to be as cross as a cat, to ride the black donkey, to bristle like a porcupine

être heureux comme un chien qui se noie

(1640)

1. cf. ci-dessus

2. être malchanceux

(E) to be dogged by misfortune

faire du chien (1867)

(cf. traîner le cheval mort)

faire le chien après qqn

suivre qqn partout

(E) to follow s.o. like a dog

faire le chien couchant auprès de qqn

1. s'humilier (1611)

2. flatter bassement (1690)

(E) 1. to cringe to s.o.

2. to eat s.o.'s toads

faire les chiens écrasés (1881)

assurer la rubrique des faits divers dans un journal

(E) to write fillers 
faire un mal de chien (1779)

être très douloureux

(E) to be awfully painful

fidèle comme un chien

d'une fidélité à toute épreuve

(E) as true as a turtle to her mate

fou comme un jeune chien (18)

étourdi, imprévoyant

(E) bird-witted, hare-brained, hen-witted

fréquenter le chien et le chat

(cf. chat)

garce à chiens (16)

(cf. araignée de trottoir)

garder un chien de sa chienne à qqn (1867)

garder rancune et songer à vengeance

(E) to have a rod in pickle for s.o.

heureux comme le chien de Brusquet, qui alla

au bois et le loup le mangea (1640)

(cf. être heureux comme un chien qui se casse le nez)

il a été à Saint-Malo, les chiens lui ont mangé

les os

il est d'une extrême maigreur

(E) he's herring-gutted

il a été mordu par un chien enragé

il est très susceptible

(E) he is very touchy

il donne l'avoine aux chiens (1640)

il se donne pour plus qu'il n'est

(E) he gives himself out for more than he is

il en est amoureux comme un chien d'un bâton (1640)

il la déteste

(E) he hates her

il est accoutumé/fait à cela comme un chien

d'aller à pied/d'aller nu tête (17)

cela lui est (devenu) habituel

(E) it has become second nature to him

il fait comme le chien de Jean de Nivelle, qui

s'enfuit quand on l'appelle (17)

il n'est pas complaisant

(E) he's not obliging

il fait comme/il ressemble les grands chiens, il

veut pisser contre les murailles (1640)

c'est au-dessus de ses forces

(E) the task is beyond his means

il mourrait plutôt un bon chien de berger (1640)

ce sont toujours les meilleurs qui meurent les premiers

(E) good men die sooner than knaves

il n'attache pas son chien/ses chiens avec des saucisses (1643)

il est d'une grande avarice

(E) he's a flea-flint, he'd skin a flea for the sake of its hide il n'en manque non plus qu'un chien de puces

(1640)

il y en a des quantités

(E) there are loads of it

il n'est chasse que de vieux chiens (1606)

rien ne vaut l'expérience de l'âge

(E) an old fox needs no craft, nothing like

the old horse for the hard road

il n'est pas bon à jeter aux chiens (1640)

c'est un individu méprisable

(E) hanging is too good for him

il ne faut point se moquer des chiens qu'on ne soit hors du village (1654)

il faut se mettre à l'abri du danger avant de

le narguer

(E) do not halloo till you are out of the wood

il ne jette pas son lard aux chiens (17)

(cf. il n'attache point son chien avec des saucisses)

il viendra un temps où les chiens auront besoin de leur queue (1690)

on peut avoir besoin un jour de gens qu'on néglige maintenant

(E) a lion may be beholden to a mouse

jamais bon chien n'aboie à faute (1640)/ à faux (1842)

un habile homme ne menace pas en vain

(E) he must have iron nails that scratches a

bear, when the old dog barks he giveth

counsel

jeter sa langue aux chiens (17)

(cf. donner sa langue au chat)

jeter un os à la gueule d'un chien pour le faire taire

acheter le silence de qqn

(E) to bribe s.o. to silence

las comme un chien

épuisé

(E) dog-tired

lavez chien, peignez chien, toutefois n'est chien que chien

(cf. un âne chargé ne laisse pas de braire)

le chien commande au chat et le chat à sa queue (1640)

(cf. chat)

(le) chien en vie vaut mieux que (le) lion mort

(cf. il vaut mieux un âne vivant qu'un savant mort)

le chien peureux n'a jamais son saoul de lard

la fortune sourit aux audacieux

(E) fortune favours the brave

les chiens aboient, la caravane passe!

faisons la sourde oreilles aux critiques

(E) the moon does not heed the barking of dogs 
les coups de bâton sont pour les chiens (cf. où la GUÊPE a passé, le moucheron demeure)

leurs chiens ne chassent pas ensemble (1690) ils ne sont pas du même bord

(E) they cannot hitch/set/stable their horses together

maigre comme un chien fou d'une extrême maigreur

(E) long and slender like a cat's elbow, as fat as a hen in the forehead/as a hen's forehead, as lean as shotten herring, herring-gutted malade comme un chien (19)

très mal(ade)

(E) as sick as a cat/dog/horse/parrot/rat

mauvais chien ne trouve où mordre

l'occasion manque toujours au maladroit

(E) clumsy persons miss every opportunity mener une vie de chien (1690)

avoir une existence difficile ou déréglée

(E) to lead a dog's life

merde de chien

(cf. c'est de la pisse d'âne)

mieux vaut un chien vivant qu'un lion mort

(cf. il vaut mieux un âne vivant qu'un savant mort)

monter à cheval comme une paire de tenailles sur le cul d'un chien (1894)

(cf. cheval)

mourir comme un chien

1. mourir dans l'abandon le plus total

2. mourir sans avoir reçu les sacrements

(E) 1. to die forsaken like a dog

2. to die without the sacraments of the church

museau de chien (1640)

musicien

(E) musician

n'être pas chien

1. être de qualité (chose) (1842)

2. être généreux (personne) (1872)

(E) 1. to be the bee's knees/the cat's pyjamas/the cat's whiskers

2. to be freehanded

nager à la chien

patauger comme les enfants

(E) to dog-paddle

nager comme un chien de plomb

être mauvais nageur

(E) to swim like a brick

ne pas donner sa part aux chiens

tenir fermement sa part

(E) to claim one's share ne réveillez pas le chien qui dort (13)

(cf. il ne faut pas réveiller le chat qui dort)

noces de chiens (17)

coït

(E) coot (US), booly- dog (US), lame duck

(US), Russian duck, bit of fish, goat's jig,

goose and duck, bit of the goose's neck,

mutton, puss(y)

nom d'un chien! (19)

(cf. ventre de biche!)

on ne peut empêcher le chien d'aboyer ni le

menteur de mentir (cf. demande-t-on à des béliers qu'ils n'aient pas de cornes?)

peau de chien (1867)

(cf. araignée de trottoir)

pendant que le chien chie/pisse, le loup s'en va (1640)

(cf. tandis que le loup chie, la BREBIS s'enfuit)

pendant que le chien pisse, le lièvre s'en va (cf. ci-dessus)

petit chien, belle queue (1640)

les hommes de petite taille seraient dotés

d'un grand sexe

(E) little men are believed to have a large penis

piquer un chien (1840)

faire un somme

(E) to catch a bird, to have a cat-nap/dognap

pleuvoir comme du chien (1867)

pleuvoir à verse

(E) to rain cats and $\underline{\operatorname{dog} \underline{s}}$, to rain chicken coops (US)

plus sot qu'un jeune chien (1640)

(cf. bête comme un âne)

pour l'alouette le chien perd son maître (1557)

(cf. alouette)

quand il rit, les chiens se battent (1640)

il rit rarement, il est souvent de mauvaise

humeur

(E) he seldon gives a laugh

quand le loup est pris, tous les chiens lui lardent les fesses (1640)

on n'a souvent aucune pitié pour celui

auquel il arrive malheur

(E) when a dog is drowning, everyone offers

him drink; when the ox falls, there are many

that will help to kill him

quand un chien se noie, tout le monde lui offre à boire (1934)

(cf. ci-dessus)

qui aime Bertrand, aime son chien (17)

il faut tout aimer en ses amis

(E) love me, love my dog 
qui hante chien, puces remporte (1597)

(cf. avec les CHIENS on ne gagne que des puces)

qui m'aime, aime mon chien (1640)

(cf. qui aime Bertrand aime son chien)

qui perd un chien et retrouve un chat, c'est toujours une bête à quatre pieds (1640)

(cf. bête)

qui se couche avec les chiens se lève avec les puces (1640)

(cf. avec les CHIENS on ne gagne que des puces)

qui veut noyer son chien l'accuse de la rage (13)

(cf. occasion trouve qui son chat bat) recevoir qqn comme un chien dans un jeu de quilles (1842)

faire mauvais accueil à qqn

(E) to welcome s.o. like a dog on a puttinggreen

ris de chien (1640)

flatterie intéressée

(E) self-interested flattery

rompre les chiens (1690)

détourner la conversation, changer de sujet

(E) to call off the dogs

rubrique des chiens écrasés (1881)

les faits divers d'un journal

(E) the accident column in a newspaper

s'accorder/s'entendre comme chien et chat (1640)

(cf. chat)

sacré-chien (1825)

(cf chien 1)

sacré nom d'un chien!

(cf. ventre de biche!)

se battre comme des chiens enragés

se battre sans merci

(E) to fight like Kilkenny cats/like rats in a corner/like tigers

se donner un mal de chien

se dépenser sans compter

(E) to take no end of pains

se regarder en chiens de faïence (19)

se regarder avec une antipathie manifeste

(E) to look at one another like stuck pigs

si vous n'avez pas d'autre sifflet votre chien est perdu

à défaut d'autres moyens, vous ne réussirez

pas

(E) one should have more than one string to one's bow

suivre comme un petit chien

(cf. suivre comme un caniche)

tandis que le chien pisse, le lièvre s'enfuit

(cf. tandis que le loup chie, la BREBIS

s'enfuit) tant l'on doit caresser le chien que l'on soit

passé (13)

(cf. il ne faut point se moquer des chiens qu'on ne soit hors du village)

tel loup, tel chien

(cf. à bon CHAT, bon rat)

temps à ne pas mettre un chien dehors (1867)

temps exécrable

(E) not a fit day out for man or beast,

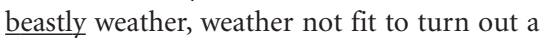
$\underline{\operatorname{dog} \text { in }}$

temps de chien (1867)

(cf. ci-dessus)

traiter qqn comme un chien (1847)

(cf. être chien avec qqn)

un chien est bien fort sur son fumier (1640)

chacun est maître chez soi

(E) a cock is bold on his own dunghill,

every $\underline{\operatorname{dog}}$ is a lion at home

un chien qui pisse fait pisser l'autre (B)

l'exemple est contagieux

(E) example is catching

un chien regarde bien un évêque (1690)

on ne doit pas s'offenser de ce qu'on vous

regarde

(E) a cat may look at a king

vieux chien est mal à mettre en lien (13)

les vieilles personnes tiennent à leur liberté

(E) old people cling to their freedom

vivre comme un chien, vivre en chien

(cf. mener une vie de chien)

votre chien mord-il encore? (1640)

êtes-vous encore fâché?

(E) are you still ratty?

\section{CHIENNE}

chienne

frange de cheveux sur le front

(E) straight fringe

chienne chaude (17)

(cf. chèvre)

chienne de vie!

la vie est dure

(E) life's a bitch!

garder un chien de sa chienne à qqn (1867)

(cf. chien)

il vaut autant être mordu d'un chien que d'une chienne (1640)

(cf. chien)

vous aurez un cochon de ma chienne (1640)

façon plaisante d'annoncer une récompense

(E) jocular way of announcing some reward 


\section{CHOUETTE}

avoir qqn à la chouette (1921)

(cf. avoir un chien pour qqn)

(c'est) chouette! (1872)

c'est splendide, magnifique, super

(E) it's the bee's knees!/the cat's pyjamas!/

the cat's whiskers!/the dog's bollocks!/the elephant's eyebrows! (US)/the goat's toe!/the mosquito's eyebrows! (US)/the pig's eye! $\underline{\text { chouette }}$

1. cf. ci-dessus (1825)

2. postérieur (1927)

(E) backside

donner/filer/refiler du chouette (1970)

être un pédéraste passif

(E) to be a bitch/gay cat $/$ chick(en)/(kid) $\underline{\text { lamb }}$

être (fait) chouette (1849)

1. cf. être le bœuf 2

2. être arrêté

(E) to be arrested

être la chouette (1842)

être l'objet habituel de moqueries

(E) to be the usual laughingstock

être larron comme chouette (17)

avoir un naturel voleur

(E) to be as thevish as a magpie

faire la chouette (1842)

jouer seul contre plusieurs

(E) to play a lone hand

porter des chouettes à Minerve

perdre sa peine, faire une besogne inutile

(E) to bring owls to Athens

prendre du chouette (1957)

être un pédéraste actif, sodomiser

(E) to be a birdie/bird-taker/bug/capon/

gooser/sheepherder/wolf

quand la chouette miaule le soir, de beau temps

on a espoir

dicton qui s'explique de soi-même

(E) the twilight hooting of the wood-owl

spells fine weather

vieille chouette (1885)

vieille femme désagréable

(E) old bitch/cat/cow

\section{CIGALE}

ferrer les cigales (1611)

perdre son temps à des choses inutiles

(E) to dog away one's time, to shoe the goose/gosling

\section{CIGOGNE}

cigogne

1. femme grande et mince

2. palais de justice, tribunal

3. bohémien (1847) (19)

(E) 1. mackerel-back

2. law courts, tribunal

3. gipsy

contes à/de la cigogne (1640)

(cf. contes de peau d'ânon)

cou de cigogne

cou long et mince

(E) long scraggy neck

dab de la cigogne (19)

procureur général

(E) attorney general

\section{CLOPORTE}

cloporte (1867)

concierge

(E) concierge, doorkeeper

cloportes en foule sur les murs, le mauvais

temps est sûr

dicton qui s'explique de soi-même

(E) throngs of wood-lice on the walls spell foul weather

\section{COBAYE}

servir de cobaye (19)

servir de sujet d'expérience

(E) to be a guinea-pig

\section{COCHON}

adieu, veau, vache, cochon, couvée!

envolées, les illusions! (17)

(E) all my swans are turned geese!

adroit comme un cochon de sa queue

très maladroit

(E) cow-handed, like a miller's mare

avoir un air cochon (1864)

avoir un air égrillard

(E) to have a goatish look

avoir un caractère de cochon

avoir très mauvais caractère

(E) to be a hell-cat/hell-dog/hell-hound

ben, mon cochon! (20)

exclamation d'étonnement

(E) well, old buck!/old dog!/old horse!/old oyster!

bouffer de la tête de cochon (19)

recevoir un coup de tête dans l'estomac

(E) to get butted in the stomach

c'est du travail de cochon

c'est un travail mal fait

(E) this job is a real pig's ear 
c'est pas cochon!

(cela n'est pas tant chien)

cochon

1. individu malpropre (17)

2. individu méprisable, salaud (17)

3. ventre (1878)

(E) 1. dirty beast/pig, filthy swine

2. beast, bee, son of a bitch, dirty dog, hog, tripehound, louse, dirty pig, pigdog, skunk, (filthy), swine, tick, tyke 3. belly

cochon (1611) (adj)

égrillard, licencieux, obscène

(E) goatish, swinish

cochon de payant (20)

1. contribuable

2. celui qui doit payer la note

(E) 1. taxpayer

2. the sucker who pays the bill

cochon qui s'en dédit! (20)

c'est une affaire conclue

(E) it's a deal!

cochonceté (1891)

propos égrillard, obscénité

(E) smutty talk, obscenity

cochonner (1808)

exécuter salement, bousiller

(E) to bitch, to make a bitch of sthg cochonnerie

1. cf. cochonceté (1668)

2. cf. tour de cochon

3. nourriture infecte

(E) dog's vomit, pigswill, pigwash

donner de la confiture aux cochons (19)

offir qqch à qqn incapable de l'apprécier

(E) to cast pearls before swine

être amis (18)/copains (1867) comme cochons

être très liés

(E) to be very close friends

être camarades comme cochons

1. cf ci-dessus (16)

2. faire souvent la noce ensemble

(E) to be as thick as thieves

être comme un cochon à l'auge

manger gloutonnement, goinfrer

(E) to eat like a horse/pig, to make a pig of oneself, to eat like a swine, to wolf it down

être fait comme un vendeur de cochons (1690)

1. être mal bâti

2. être mal habillé

(E) 1. to be misshapen

2. to be dressed up like a scarecrow/a dog's dinner gras comme un cochon (17)

très gros

(E) as fat as a pig

il deviendra qqn si les petits cochons ne le mangent pas (19)

la réussite dépend des aléas de la vie

(E) success depends on the hazards of life

il faut mourir, petit cochon, il n'y a pas plus

d'orge (1640)

les ressources sont épuisées

(E) all resources are used up

jouer un pied de cochon à qqn (19)

(cf. brider la bécasse)

jouer un tour de cochon à qqn (1898)

se comporter très mal avec qqn, nuire à qqn

(E) to give s.o. the dog to hold, to play s.o. a lousy trick

l'avare et le cochon ne sont bons qu'après leur mort (1842)

on n'en tire rien de leur vivant

(E) like a pig, no good alive

le cochon qui sommeille en tout homme

les mauvais instincts, la sexualité

(E) the sexual instincts present in every man

les cochons de son âge ne sont plus bons à rôtir (1640)

l'âge est là

(E) he's no spring chicken, the black ox has trod on his foot

manger comme un cochon

(cf. être comme un cochon à l'auge)

manger le cochon ensemble (17)

s'entendre pour tromper autrui

(E) to contrive together to dupe s.o.

mener une vie de cochon (17)

ne songer qu'à manger et à dormir

(E) to think of nothing but food and sleep

mieux vaut cochon qui s'en dédit que cochon

qui sent les pieds (20)

il vaut encore mieux avoir à faire à qqn qui ne tient pas sa promesse qu'à qqn qui pue

des pieds

(E) smelly feet are worse than a breech of promise

ne pas savoir si c'est du lard ou de cochon (19)

(cf. ne pas savoir si c'est BIQUE ou bouc)

nous n'avons jamais gardé les cochons ensem-

ble! (1865)

cette familiarité est déplacée

(E) don't be so familiar!

on n'engraisse pas les cochons avec de l'eau claire

(cf. à chair de loup, dent de CHIEN)

on ne peut pas avoir le lard et le cochon

on ne peut pas avoir tout à la fois

(E) you cannot eat your cake and have it 
pied de cochon (1872)

(cf. basset 2)

plein comme un cochon

(cf. saoul comme un âne)

rappeler le cochon (17)

(cf. reprendre sa chèvre à la barbe)

rince-cochon

verre d'eau minérale bu après une cuite

(E) glass of mineral water after a binge

sale comme un cochon

malpropre, dégoûtant

(E) as filthy as a pig

saoul comme un cochon (1690)

repu d'avoir goinfré

(E) as full as a tick

soigner son cochon (1878)

bien manger

(E) to have a good tuck-in

temps de cochon

(cf. temps à ne pas mettre un chien dehors)

tête de cochon

(cf. âne 3)

tirer un pied de cochon à qqn (1894)

(cf. jouer un tour de cochon à qqn)

tour de cochon (19)

mauvais tour, mystification

(E) dog-trick, lousy trick, monkey business

travailler comme un cochon (19)

bâcler son travail, faire qqch maladroitement

(E) to make a bitch/a dog's dinner/a pig's

ear/a sow's ear of sthg

un cochon n'y trouverait pas ses petits (19)

(cf. une chatte n'y retrouverait pas ses jeunes)

vieux cochon

vieillard licencieux

(E) filthy old beast, dirty old dog

vous aurez un cochon de ma chienne (1640)

(cf. chienne)

yeux de cochon (16)

petits yeux ronds

(E) pig eyes

\section{COCKER}

avoir les roberts en oreilles de cocker (1953)

avoir des seins flasques

(E) to have flabby breasts

\section{COLIBRI}

colibri

1. jeune fille pauvre (1894)

2. individu indigne de confiance (1935)

(E) 1. poor girl

2. son of a bitch, louse, rat, skunk

\section{COLOMBE}

à colombe saoule les cerises sont amères (14) quand on est repu on dédaigne les choses les meilleures

(E) when one is satiated the best things are no longer tempting

à la colombe solitaire les cerises sont amères la vieille fille n'a pas une vie agréable

(E) an old maid's life is not a pleasant one colombe

1. cf. biche 3 (1850)

2. cf. araignée de trottoir (1887)

colombe de Vénus (19)

(cf. chat 2)

craignez la colère de la colombe (1842)

(cf. quand les BREBIS enragent elles sont pires que les loups)

l'aigle n'engendre pas la colombe (1842) (cf. aigle)

la bave du crapaud n'atteint pas la blanche colombe!

(cf. les chiens aboient, la caravane passe)

les colombes et les faucons (1960)

les pacifistes et les bellicistes

(E) the doves and the hawks

ma colombe!

(cf. ma biche)

\section{CONGRE}

aller aux congres sans crochet (1842)

entreprendre une affaire sans avoir les

moyens de l'exécuter, se montrer

imprévoyant

(E) to go rabbit-hunting with a dead ferret

\section{COQ}

au chant du coq (17)

(cf. au chant de l'alouette)

avoir les mollets de coq (1867)

(cf. avoir la jambe tout d'une venue comme

la jambe d'un chien)

avoir les deux coqs (1939)

être âgé de quarante ans

(E) to be forty

bon coq (1864)

(cf. âne débâté)

chanter le coq (1640)

parler plus fort que son mari

(E) to talk louder than one's husband

chétive est la maison où le coq se tait et la poule chante

un ménage ne peut être prospère si le mari obéit et la femme commande

(E) it's a sad house where the hen crows louder than the cock 
coq

1. cf. bon coq

2. chef (18)

3. champion (1833)

4. œil poché (19)

5 . pièce de vingt francs en or (1903)

(E) 2. boss, chief

3. top-dog

4. mouse

5. 20-franc gold coin

coq-à-l'âne

(cf. âne)

coq chante ou non, viendra le jour (1597)

l'inéluctable n'a pas besoin de prophètes

(E) what is ineluctable needs no prophesying

deux coqs vivaient en paix, une poule survint... cherchez la femme!

(E) there's a woman in it !

être/vivre comme un coq en pâte (1672)

mener une vie douillette, être bien aise, ne manquer de rien

(E) to be like a bird on a tree, to be as snug as a bug in a rug, to live like a fighting cock, to be as warm as a mouse in a churn/as snug as a pig in pease-straw, to be like pigs in clover

faire boire le coq

faire des ricochets sur l'eau

(E) to play at ducks and drakes

faire des coqs-à-l'âne (1842)

(cf. âne)

fier comme un coq (17)

très fier, satisfait

(E) all cock-a-hoop, as proud as a $\underline{\operatorname{dog}}$ in a doublet/as old Cole's $\underline{\text { dog/ as a peacock }}$

hardi comme un coq sur son fumier se sentant fort parce qu'on est chez soi

(E) as bold as a cock, like a cock on his own dunghill

la maison est à l'envers lorsque la poule chante aussi haut que le coq (1585)

(cf. chétive est la maison où le COQ se tait et la poule chante)

la poule ne doit pas chanter devant le coq (17) (cf. ci-dessus)

le coq du quartier (1640) / du village (1842) homme qui séduit toutes les belles du lieu

(E) the cock of the roost/walk

les bons coqs sont toujours maigres (1498)

les hommes ardents aux plaisirs amoureux ne sont jamais gros

(E) men who indulge in the pleasures of the flesh are seldom fat où la bonne femme vendit au coq (17)

lieu indéterminé

(E) undetermined place

poussin chante comme le coq lui apprend (13)

les enfants ont besoin de modèles

(E) dogs bark as they are bred, the young

pig grunts like the old sow

quand en été le coq boit, la pluie est au-dessus des toits

dicton qui s'explique de soi-même

(E) when the cock drinks in the summer,

rain is not far off

quand le coq chante à la veillée, il a déjà la

queue mouillée

dicton qui s'explique de soi-même

(E) when the cock crows in the evening,

rain is coming

quand le coq chante à midi, signe d'un temps de paradis

dicton qui s'explique de soi-même

(E) when the cock crows at noon, heavenly

weather soon

quand le coq chante avant la nuit, signe de pluie

(cf. quand le coq chante à la veillée, il a déjà

la queue mouillée)

quand le coq chante le soir, c'est signe qu'il va

bientôt pleuvoir

(cf. ci-dessus)

quand le coq chante souvent, signe d'un

changement de temps

dicton qui s'explique de soi-même

(E) a cock crowing all the time heralds a weather change

renier au premier chant du coq

abandonner au premier signe de danger

(E) to give up at the first sign of danger

rouge comme un coq (1372)

le visage tout rouge (de honte, de colère, etc)

(E) as red as a boiled lobster/a turkey-cock

sauter du coq à l'âne (15)

(cf. âne)

se lever au chant du coq (17)

se lever de bonne heure

(E) to be an early bird, to rise at cock-crow,

to rise with the lark

si entre trois et quatre heures le coq a chanté, le temps est gâté

dicton qui s'explique de soi-même

(E) if the cock crows between 3 and 4 p.m.

the weather will be breaking up

si le coq chante avant minuit, c'est du brouillard ou de la pluie

(cf. quand le coq chante à la veillée, il a déjà

la queue mouillée) 
un bon coq n'est jamais gras

(cf. les bons coqs sont toujours maigres)

un coq est bien fort sur son fumier (1749)

(cf. hardi comme un coq sur son fumier)

\section{CORBEAU}

aile de corbeau (19)

noir intense

(E) raven-black

bec-de-corbeau

1. outil tranchant à extrémité recourbée

2. pince à fil de fer

(E) 1. rave-hook

2. wire nippers

corbeau

1. employé des pompes funèbres

2. prêtre en soutane (1845) (1867)

3. homme acharné au gain (1882)

4. auteur de lettres anonymes

(E) 1. undertaker's mute

2. dog-collar

3. rapacious man

4. anonymous writer of poison-pen letters

coucher de poule et lever de corbeau écartent l'homme du tombeau

il est bon pour la santé de se coucher tôt et de se lever de même

(E) go to bed with the lamb and rise with the lark

de mauvais corbeau, mauvais œuf (1640)

la méchanceté engendre la méchanté

(E) a wild goose never laid a tame egg

être ravitallé par les corbeaux (20)

habiter loin de tout

(E) to live in a remote place

fourrage de corbeaux (1640)

malfaiteurs pendus

(E) gallows birds

jamais corbeau n'a fait un canari

(cf. l'AIGLE n'engendre pas la colombe)

les corbeaux ne crèvent pas les yeux aux

corbeaux (1568)

les gens de même espèce ne se nuisent pas

entre eux

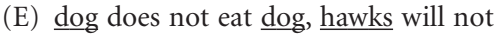
pick hawks' eyes out

noir comme un corbeau (19)

très noir

(E) as black as a crow/as a raven's wing, raven-black

nourris un corbeau, il te crèvera l'œil/les yeux le méchant rend le mal pour le bien

(E) breed up a crow and he will tear out your eyes nul lait noir ni blanc corbeau (1557)

la nature est immuable, le monde est conservateur

(E) nature does not change, the world is conservative

se disputer un héritage comme des corbeaux se le disputer âprement

(E) to fight like vultures over an inheritance

\section{CORMORAN}

cormoran

1. cf. corbeau 1 (1797)

2. homme sec et maigre (1690)

3. Juif (1960)

(E) 2. herring-gutted fellow, mackerel-back 3. eagle-beak

\section{CORNEILLE}

bayer aux corneilles (17)

ne rien faire, flemmarder

(E) to catch flies, to chase mice

béer aux corneilles (1960)

1. cf. ci-dessus

2. cf. s'embêter comme une carpe

ce que chante la corneille, ainsi chante le cornillon (15)

(cf. poussin chante comme le COQ lui apprend)

la corneille d'Ésope (1690)

auteur qui dérobe les idées des autres

(E) author who appropriates the ideas of others

quand les corneilles s'assemblent, du bois pour ton hiver assemble

dicton qui s'explique de soi-même

(E) when the crows flock together, prepare

for wintry weather

y aller de cul et de tête, comme une corneille qui abat des noix (1690)

$y$ aller de toutes ses forces

(E) to go the whole animal $/ \underline{h o g} / \underline{\text { swine, }}$, to go ahead like a whale

\section{COUCOU}

au temps où chante le coucou, le soir sec,

le matin mou

au printemps, le temps est encore instable

(E) when the cuckoo sings in the spring the weather is still changeable

avaler comme un coucou (1842)

(cf. être comme un cochon à l'auge)

avoir une vraie pine de coucou (1953)

être doté d'un très petit pénis

(E) to have very small bird 
coucou

1. cf. bélier

2. montre (1828); pendule

3. chapeau melon (1899)

4. petit avion (1914)

(E) 2. bull's eye, clickman toad, cuckoo clock

3. bowler hat

4. flying flea

coucou! (1887)

interjection pour marquer sa présence

(E) bo-peep!, peekaboo!

être (fait) coucou (1690)

être trompé par sa femme

(E) to get the bull's feather, to go through the ox-house to bed

faire coucou (1867)

jouer à se cacher

(E) to play at peekaboo

faire coucou à un homme (1865)

le tromper avec sa femme

(E) to give the bull's feather

la barbe d'orge coupe au coucou la gorge

à la saison de l'orge, le coucou ne chante plus

(E) when the barley season comes the cuckoo voiceless becomes

le coucou avant les feuilles, beaucoup de paille mais peu de grain

si l'arrivée du coucou coïncide avec un

printemps précoce, le blé montera en tige et

fournira peu de grain

(E) if the cuckoo arrives with an early

spring, straw will be plenty but wheat scarce

maigre comme un coucou (1842)

(cf. maigre comme un chien fou)

quand chante le coucou, le matin mouillé, le soir séché

(cf. au temps où chante le coucou, le soir sec, le matin mou)

quand le coucou arrive déshabillé, peu de paille, beaucoup de blé

si l'arrivée du coucou coïncide avec un printemps tardif (sans feuilles), le blé ne monte pas haut sur tige et fournira beaucoup de blé

(E) if the cuckoo comes with a late spring, straw will be scarce and wheat plenty

un jour est mouillé, l'autre sec, quand le coucou ouvre son bec

(cf. au temps où chante le coucou, le soir sec, le matin mou)

\section{COULEUVRE}

avaler des couleuvres

1. subir des affronts (1667)

2. éprouver des déceptions (1867)

3. croire des calembredaines (1878)

(E) 1. to eat crow

2. to meet with disappointments

3. to swallow cock-and-bull stories

couleuvre

1. personne perfide (12)

2. affront (16)

3. mensonge, sornette

(E) 1. falsehearted person

2. insult

3. cock, horseshit

faire avaler des couleuvres à qqn

1. cf. faire avaler des anguilles à qqn (19)

2. faire des affronts à qqn (17)

(E) to make s.o. eat crow

paresseux comme une couleuvre (20)

extrêmement paresseux

(E) as lazy as Ludlam's dog (that leaned its

head against a wall to bark)

quand la couleuvre traverse le chemin, orage avant demain matin

dicton qui s'explique de soi-même

(E) a grass snake crossing the path spells a storm before next morning

\section{COURLIS}

sous grand vent mâture geindra quand courlis volant chantera

si l'on entend le courlis crier en vol, il faut s'attendre à grand vent

(E) expect fierce wind when you hear the flying curlew shriek

\section{CRABE}

crabe

1. cf. canard 4

2. cf. chameau 1 (1901)

3. cf. chat 3 (1970)

4. comédien médiocre (1916)

5. pou de pubis (1928)

6. porte-monnaie (1928)

7. cancer (1975)

(E) 4. second-rate actor

5. crablouse

6. purse

7. cancer

face de crabe! (20)

(cf. chameau 2)

fainéant comme un crabe

(cf. paresseux comme une couleuvre) 
marcher en crabe (20)

se déplacer de travers

(E) to walk crabwise

panier de crabes (1942)

groupe d'ambitieux qui cherchent à s'évincer l'un l'autre

(E) rat race

vieux crabe (1890)

vieillard rendu hargneux par l'âge

(E) pig-headed old fool

\section{CRAPAUD}

avaler un crapaud (19)

faire qqch qui coûte beaucoup à son auteur

(E) to do sthg that costs its author a lot

avaleur de crapauds (19)

(cf. bouc émissaire)

baver comme un crapaud

calomnier

(E) to give green rats

constipé du crapaud (1948)

(cf. chien adj.)

crapaud

1. cf. sale bête 1 (1541)

2. cf. crabe 6 (1899)

3. cadenas (15)

4. petit garçon (1866)

(E) 3. padlock

4. chick, mite, urchin

en être chargé/pourvu comme un crapaud de plumes (17)

être complètement dépourvu de qqch

(E) to be as full of it as a toad is of feathers

faire crapaud (1881)

boire en solitaire

(E) to drink with the flies

faire un oil de crapaud mort d'amour (1953)

(cf. faire des yeux de carpe frite)

il fera beau temps, les crapauds sautent (1640)

se disait ironiquement quand on voyait un

lourdaud se mettre à sauter

(E) jocular way of predicting faire weather when a lumpish fellow was seen jumping

il n'y a pas de grenouille qui ne trouve son

crapaud

à chacun sa chacune

(E) every Jack has his Jill

la bave du crapaud n'atteint pas la blanche colombe

(cf. les chiens aboient, la caravane passe)

laid comme un crapaud

très vilain

(E) as ugly as bull-beef quand les crapauds chantent, le beau temps

s'avance

dicton qui s'explique de soi-même

(E) the croaking of toads spells fine weather

regarder qqn avec des yeux de crapaud mort

d'amour (20)

(cf. faire des yeux de carpe frite)

saute, crapaud, voici la pluie! (1640)

(cf. il fera beau temps, les crapauds sautent)

sauter comme un crapaud (1690)

faire qqch à contre-cœur

(E) to do sthg reluctantly

\section{CREVETTE}

crevette

1. cf. araignée de trottoir (1866)

2. cf. biche 2 (1866)

3. jeune homosexuel, prostitué occasionnel (1983)

(E) young homosexual, occasionally a prostitute

môme crevette

(cf. asticot 4)

\section{CROCODILE}

crocodile

1. créancier (1866)

2. usurier (1866)

3. individu intraitable en affaires (1866)

4. malfaiteur chevronné (1975)

(E) 1. ornithorhynchus

2. shark, wolf

3. shark, wolf

4. experienced criminal

larmes de crocodile (1562)

douleur feinte

(E) crocodile tears

\section{CYGNE}

blanc comme un cygne

d'une éclatante blancheur

(E) as white as snow

chant du cygne

1. œuvre ultime (1546)

2. dernier sursaut amoureux avant la

vieillesse (17)

(E) swan song

chanter le chant du cygne (1640)

être à l'article de la mort

(E) to be on the verge of death

faire un cygne d'un oison

faire de qqn un éloge exagéré, injustifié

(E) to consider all one's geese swans 
foutre un cygne (19)

coïter, l'homme à genoux et les jambes de la femme sur ses épaules

(E) to copulate with the woman's legs on the shoulders of the kneeling man

le cygne, plus il vieillit, plus il s'embellit (1612) une chevelure blanche embellit l'homme

(E) white hair improves the looks of a man on ne voit cygne noir ni nulle neige noire (1568)

(cf. nul lait noir ni blanc corbeau) pousser le boum! du cygne (19)

(cf. mettre la table pour les asticots)

\section{DAIM}

daim (19)

1. cf. âne 2

2. jeune élégant

(E) buck

daim huppé (1835)

homme riche et vaniteux, bon à duper

(E) goldfinch

puer comme un daim

(cf. puer comme un bouc)

\section{DAUPHIN}

dauphin

1. cf. barbeau (1866)

2. pince à effraction (1821)

(E) burglar's jemmy

\section{DINDE}

(petite) dinde (1752)

(cf. bécasse)

plumer la dinde

1. tirer de l'argent de qqn qui se laisse

dépouiller sottement

2. jouer de la guitare (1982)

(E) 1. to flap a jay, to skin the lamb, to pluck

a pigeon

2. to play the guitar

se bourrer la dinde (1928)

se garnir pendant les règles

(E) to pad oneself during the menses veillir dinde

(cf. vieille bique)

\section{DINDON}

aller garder les/ses dindons (1690)

prendre sa retraite, se retirer à la campagne

(E) to be gone to raise chickens (US)

bête comme un dindon

(cf. bête comme un âne)

c'est la danse des dindons (1842)

impression que l'on donne de faire de bonne grâce qqch à contre-cœur

(E) giving an impression of willingly doing sthg one is reluctant to do

colère comme dindon

très en colère

(E) as mad as a buck/as Barney's bull, ratty dindon

1. homme vaniteux et niais (1793)

2. jésuite (1830)

(E) 1. conceited, silly man

2. Jesuit

être le dindon de la farce (1835)

(cf. être le bœuf 2)

nous n'avons pas gardé les dindons ensemble!

(cf. nous n'avons jamais gardé les cochons ensemble!)

\section{DOGUE}

dogue (1536)

(cf. bouledogue 2)

être d'une humeur de dogue (1806)

(cf. être d'une humeur de chien)

être saoul comme un dogue (1690)

(cf. saoul comme un cochon)

gros dogue (1690)

homme rébarbatif (qui garde une entrée)

(E) big dog

\section{DORYPHORE}

doryphore (1940-45)

soldat allemand

(E) German soldier

(à suivre) 